\title{
The Influence of Exercise and Caffeine on Cognitive Function in College Students
}

\author{
Rachel J. Shulder, Eric E. Hall, Paul C. Miller \\ Department of Exercise Science, Elon University, Elon, USA \\ Email: ehall@elon.edu
}

Received 16 December 2015; accepted 25 January 2016; published 28 January 2016

Copyright (C) 2016 by authors and Scientific Research Publishing Inc.

This work is licensed under the Creative Commons Attribution International License (CC BY). http://creativecommons.org/licenses/by/4.0/

(c) (i) Open Access

\begin{abstract}
Exercise has widely been shown to improve cognition, potentially by making individuals more receptive to sensory stimulation or inhibiting irrelevant information. Caffeine, one of the world's most widely used stimulants, seems to have similar effects. It seems that both exercise and caffeine improve cognitive function separately, but little research has been done examining their combined effects. The purpose of this study was to examine the impact of caffeine and exercise, independently and combined, on cognitive function. 20 healthy college students completed the study. These participants were low caffeine consumers. Each participant came to the lab 5 times. During the first session, they completed a graded exercise test on a cycle ergometer to determine ventilatory threshold (VT). The following four sessions were test sessions involving supplementation and exercise. During these, each participant engaged in 30 minutes of cycling (at $90 \% \mathrm{VT}$ ) or 30 minutes of quiet reading after consuming either caffeine (at $4 \mathrm{mg} / \mathrm{kg}$ body weight) or a placebo. The Contingent Continuous Performance Task (CPT) and Wisconsin Card Sorting Task were used to measure cognitive function and were completed 5 minutes and 20 minutes after exercise or quiet reading. There were no significant differences found for any variables tested, for condition effect, time effect or condition*time interaction, except for a significant time effect on false alarms on the Contingent CPT ( $p=0.017)$. This study may have been limited by multiple variables including the population, executive function measures, caffeine dosage, or exercise prescription. These findings point to the need for future research to understand the changes in cognition from exercise and caffeine in combination. Future research may include looking at exercise at different intensities, different dosages of caffeine, or looking at the long-term cognitive effects.
\end{abstract}

\section{Keywords}

Exercise, Caffeine, Cognitive Function, Executive Function 


\section{Introduction}

Exercise has widely been shown to improve cognition by making individuals more receptive to sensory stimulation or inhibiting irrelevant information. Changes include increased long-term and short-term memory, increased executive function and decreased reaction time [1] [2]. Moderate steady-state exercise increases central nervous system arousal, which may make the individual more receptive to stimulation and increasing motor process speeds [2]. It is also possible that with limited resources (oxygen, neurotransmitters, etc.), resources are directed only to important brain centers, allowing us to focus on those tasks [3]. Pontifex, Hillman, Fernhall, Thompson, and Valentini [4] studied the effect of a 30-minute bout of running (at $60 \%-70 \% \mathrm{VO}_{2} \mathrm{max}$ ) on working memory, an aspect of executive control, in 21 young adults. Reaction time and accuracy were measured, and the results indicated shorter reaction time both immediately after exercise and 30 minutes after completion of exercise. Reaction time was fastest after exercise during the tasks requiring increased working memory capacity. These results are consistent with a recent meta-analysis of the effect of acute exercise on cognitive performance [5].

In addition to exercise, caffeine is suggested to have positive effects on multiple aspects of cognition including vigilance, mental alertness, reaction time, visual selective attention, task switching, conflict monitoring and response inhibition [6]-[8]. Caffeine is thought to cause these improvements by antagonizing adenosine receptors; it blocks the action of this inhibitory neurotransmitter by directly acting on both pre- and postsynaptic receptors [8]. Caffeine antagonizes the adenosine receptors in dopamine-rich brain areas, resulting in increased wakefulness and motor activity. Childs and de Wit [7] investigated the effects of 0, 50, 150 or 450 mg caffeine on light, nondependent caffeine users (to rule out the possibility of caffeine's effects being due to reversal of withdrawal). Forty minutes after ingestion, allowing time for plasma levels of caffeine to peak, participants completed tasks including attention, short-term memory and inhibition. Participants consuming caffeine increased the number of correct response and decreased reaction time, though memory was impaired. The researchers concluded caffeine has psychoactive effects, enhancing performance in consumers, dependent on dose and nature of the task.

Hogervorst and colleagues [9] provide one of the few studies which examined the combined effects of exercise and caffeine on measures of cognitive function. In this study, fifteen young male athletes were given a water placebo, a carbohydrate-electrolyte placebo, or carbohydrate-electrolyte solutions containing $150 \mathrm{mg}, 225$ mg or 320 mg caffeine. A cognitive battery, including attention, psychomotor speed, visual detection, and longterm memory tasks; was completed before and immediately after a 1-hour cycling bout. After exercise, all cognitive functions were improved in the participants who had consumed the beverages with $150 \mathrm{mg}$ and $225 \mathrm{mg}$ caffeine. This novel finding, showing the effects of caffeine on cognitive performance after exercise, demonstrated an improvement in complex cognitive functions, dependent on dose. A follow-up study showed similar improvement: better concentration, faster response speed and detection and improved scores on RVIP, Stroop and Visual Search tasks after caffeine ingestion and an exercise bout compared to placebo ingestion [10].

It seems that both exercise and caffeine improve cognitive function separately, but little research has been done examining their combined effects. The purpose of this research is to determine the effect that caffeine and aerobic exercise have on executive function in young adults. More specifically, this study looked at the effects of exercise + caffeine, exercise + no caffeine, no exercise + caffeine, no exercise + no caffeine on executive function. Based on previous research, it was hypothesized that performance would be the best during the exercise + caffeine session, and performance would be worst during the no exercise + no caffeine session.

\section{Methods}

\subsection{Participants}

Twenty healthy undergraduate college students (16 females, 4 males) were recruited for this study. Recruitment of participants occurred through a posting on the university's website as well as flyers posted in classrooms at the university. All participants reported they were in good health and free of any medical conditions or injuries that may be contraindications of aerobic exercise. All participants completed a caffeine intake log and a written informed consent form which was approved by the university’s Institutional Review Board. Participants were compensated with a \$25 gift-card. See Table 1 for participant descriptive information.

\subsection{Measures}

The modified Wisconsin Card Sort Task (WCST) and Contingent Continuous Performance Task (CPT) were 
Table 1. Participant characteristics (Means \pm SD).

\begin{tabular}{ccc}
\hline & Males & Females \\
\hline Age (years) & $19.3 \pm 0.5$ & $19.9 \pm 1.2$ \\
Height (in) & $71.4 \pm 1.9$ & $64.1 \pm 2.8$ \\
Weight (lbs) & $150.4 \pm 12.1$ & $143.6 \pm 22.5$ \\
Body Fat (percent) & $13.9 \pm 2.6$ & $27.5 \pm 5.2$ \\
BMI (kg/m²) & $20.7 \pm 0.9$ & $24.7 \pm 4.4$ \\
$\mathrm{VO}_{2}$ peak (mL O $\left./ \mathrm{kg} \cdot \mathrm{min}\right)$ & $39.7 \pm 8.5$ & $29.4 \pm 6.3$ \\
$\mathrm{Caffeine} \mathrm{Intake} \mathrm{(mg/day)}$ & $42.5 \pm 79.1$ & $114.4 \pm 88.6$ \\
\hline
\end{tabular}

administered using Neuroscan's STIM2 software (Compumedics Limited; Australia). The tests were projected onto a 17" computer screen in front of the participant. A 4-buttoned Compumedics Neuroscan-Stim System Switch Response Pad (Compumedics Limited; Australia) was used to record participant responses for each of the tasks.

A modified version of the Wisconsin Card Sort Task (WCST) was used in the present study. The WCST was designed to test abstract behavior and shift of set. It has been shown to be an effective measure of executive function, assessing multiple dimensions of cognition including cognitive processing speed, concept formation, inhibition capacity and cognitive flexibility [11].

The task consists of 64 stimulus cards and 4 base cards. The four base cards were always one red triangle, two yellow stars, three green crosses and four blue circles, displayed from left to right at the top of the screen. Each stimulus card had one of the four shapes (triangle, star, cross, or circle), colors (red, yellow, green, or blue) and number of objects (one, two, three, or four). The stimulus cards were displayed in the bottom right corner of the screen. The participant was told by the researcher to match the stimulus card to one of the four base cards by color, shape or number. Feedback was given as to whether the card matching was correct by a tone and the appearance of the word "CORRECT" or "INCORRECT" at the top of the screen. Participants were unaware, but the computer changed the rule after ten cumulative correct placements of the cards. The test was completed after the participant successfully sorted 60 cards. The participants were allowed as long as was necessary to finish the task. Total errors from each trial were recorded as a measure of executive control. Total errors were calculated as the number of times a card was matched incorrectly based on the currently applicable rule.

The Contingent Continuous Performance Task (CPT) is an attentional task involving executive control components. To successfully complete the CPT task, inhibitory control, a part of executive control processes, must prevent mistakes [12]. The participant was presented with a series of letters flashed in the middle of the screen for $200 \mathrm{~ms}$, with $1000 \mathrm{~ms}$ in between each letter. The instructions were to press the first button on the response pad when the target letter " $T$ " was shown. The letter "S" always preceded the letter "T" but the display of " $\mathrm{S}$ " did not mean that the target letter "T" would necessarily follow. A lure occurred when the letter "S" was shown and the letter " $\mathrm{T}$ " did not follow. Twenty target letters and ten lures were presented in each trial of the task. Average reaction time (RT) to each target letter was recorded. A false alarm (FA) was recorded anytime the participant pressed the button and the target letter "T" was not shown.

\subsection{Procedures}

All participants came into the lab for five sessions. The participants completed 1 session per day with a minimum of 48 hours between sessions. All sessions took place at the same time of day. During the first session, participants completed the informed consent form and height and weight were assessed. The participant was fitted with a Polar Target heart rate monitor and a V2 mask and headstrap (Hans Rudolph, Inc; USA). The participants were seated on a Corival Lode recumbent bike (Lode B.V. Technology; Groningen, The Netherlands) and a breathing mask was attached to record expired oxygen and carbon dioxide levels using a Parvo Medics True One Metabolic System. After a 60 second warm-up at $0 \mathrm{~W}$, the exercise test began with two minutes at $50 \mathrm{~W}$ and increased $25 \mathrm{~W}$ every two minutes until volitional exhaustion. Heart rate, $\mathrm{VO}_{2}$ and Respiratory Exchange Ratio (RER) were constantly recorded throughout the trial and participants were verbally encouraged to go as 
long as possible. At the end of the trial, the breathing mask was taken off and the participant was told to continue biking at $0 \mathrm{~W}$. Expired gas data was used to determine $\mathrm{VO}_{2}$ peak and ventilatory threshold (VT). VT was determined in order to set individualized exercise intensity for each participant in the following sessions. VT is the point during graded exercise when ventilation rate increases exponentially while workload continues to increase linearly.

Sessions two through five measured cognitive function after one of four conditions: exercise + caffeine, exercise + no caffeine, no exercise + caffeine, no exercise + no caffeine. The order of sessions were randomly assigned and counterbalanced so each participant took part in each of the conditions, and each session lasted eighty to ninety minutes total. Participants were told after the first session to arrive for each following session dressed in workout clothes and sneakers, ready to exercise if necessary, and ready with reading material of their choice if not asked to exercise. Participants were also asked to abstain from both caffeine and exercise for a minimum of 12 hours before testing sessions. Immediately on arriving to the lab for sessions two through five, the participant was given a colored capsule containing anhydrous caffeine ( $4 \mathrm{mg} / \mathrm{kg}$ body weight; NutraBio Pharmaceutical Grade Caffeine) or placebo (Arrowhead Mills Organic Brown Rice Powder). This study was double blind so the participants and first author were unaware of the contents of the capsules consumed. The capsules content was revealed upon completion of the study. Upon arriving to the laboratory for the second session, or first cognitive testing session, participants were introduced to the cognitive tests and allowed to practice one trial each of WCST and CPT. Body composition (BMI and percentage body fat) was measured at the second session by bioelectrical impedance using a OMRON Fat Loss Monitor (OMRON Healthcare Inc; Bannockburn, Illinois).

After consuming the capsule at the start of each session, the participant was fitted with a heart rate monitor and was told to sit quietly for thirty minutes. They were allowed to read, or use a computer or other technology, but not allowed to leave the laboratory. Resting HR was recorded at the end of the thirty-minute delay. During sessions with exercise, the participant was seated on a SciFit recumbent bike (SCIFIT Systems Inc.; Tulsa, OK) and told to start pedaling. After a minute of warm-up at self-determined power, the power was increased to elicit $90 \% \mathrm{VT}$ of the participant. Ten minutes into exercise, twenty minutes and thirty minutes (immediately before the end of exercise), HR was recorded to validate the participant was exercising at the proper intenisty. Water was offered every ten minutes but participants could choose whether or not they wanted to drink. After the exercise bout, the participant was told to dismount, offered additional water, and waited five minutes to complete the WCST and CPT. The WCST was always performed first, followed by the CPT. The participants then waited another fifteen minutes (for a total of twenty minutes post-exercise) and completed the WCST and CPT again.

During sessions with no exercise, the participants completed the same protocol, but instead of cycling for thirty minutes, they were simply asked to stay seated on the SciFit recumbent bike and quietly read for thirty minutes. The reading material was the participant's choice. No music or technology was allowed during this thirty-minute session. After completing the thirty minutes, participants waited five minutes and completed the cognitive test battery. They then waited until twenty minutes post-exercise and completed the second round of the cognitive tests.

\subsection{Statistical Analyses}

To determine if there was a significant difference between the different conditions and over time for the dependent variables (cognitive function), a 4 (Condition: caffeine + exercise, caffeine + no exercise, exercise + no caffeine, no caffeine + no exercise) by 2 (Time: 5 min post, 20 min post) Repeated Measures General Linear Model (RM GLM) was performed. Separate RM GLM analyses were run for reaction time and false alarms from the Contingent CPT as well as for total errors from the WCST. A p value of $<0.05$ was used to determine if the condition, time or condition by time interaction were significant.

\section{Results}

The 4 (Condition: caffeine + exercise, caffeine + no exercise, exercise + no caffeine, no caffeine + no exercise) by 2 (Time: post 5, post 20) RM GLM did not reveal a significant condition effect $[F(3,17)=2.80, p=0.071]$, time effect $[F(1,19)=2.47, \mathrm{p}=0.133]$ or condition*time interaction $[F(3,17)=2.36, \mathrm{p}=0.108]$ for reaction time on the Contingent CPT. These results indicate that there was no differences between conditions or over time for reaction time (See Figure 1). 


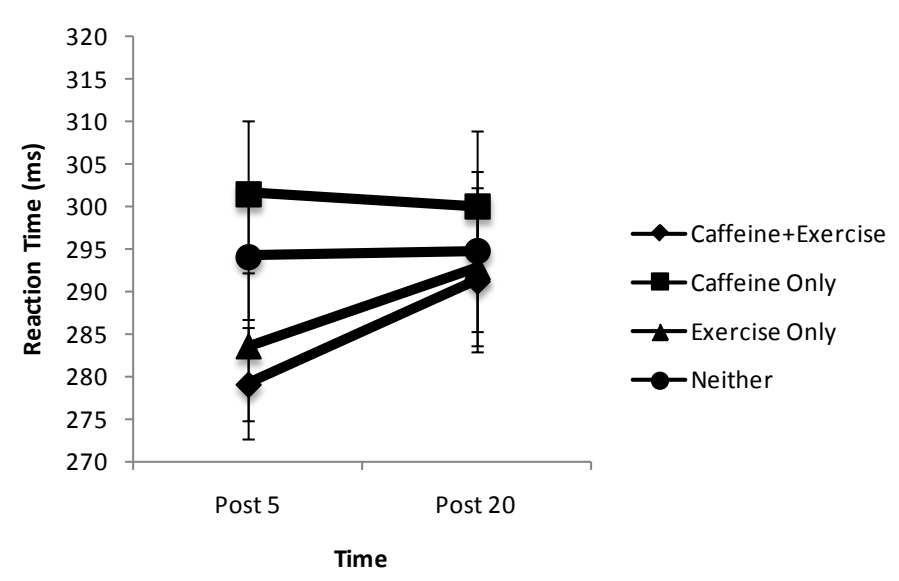

Figure 1. Reaction time for contingent CPT by condition*time.

For false alarms on the Contingent CPT a 4 (Condition) by 2 (Time) RM GLM did not reveal a significant condition effect $[F(3,17)=1.85, \mathrm{p}=0.176]$ or condition $*$ time interaction $[F(3,17)=1.69, \mathrm{p}=0.206]$ but did reveal a significant time effect $[F(1,19)=6.83, \mathrm{p}=0.017]$. This suggests that on the second administration of the test (20 minutes post condition) participants performed better as indicated by fewer false alarms on the Contingent CPT (See Figure 2).

The RM GLM for total errors on the WCST did not show a significant condition $[F(3,17)=0.41, \mathrm{p}=0.752]$, time $[F(1,19)=2.25, \mathrm{p}=0.150]$ or condition $*$ time interaction $[F(3,17)=1.09, \mathrm{p}=0.380]$. These results demonstrate that there were no statistical differences in performance across the different conditions or over time on total errors of the WCST (See Figure 3).

\section{Discussion}

The purpose of this study was to examine the effect of caffeine and aerobic exercise, together and separately on executive function. The current study failed to show significant differences between the four groups on any of the measures of cognitive function.

The current study showed no significant differences between conditions' reaction times, in contrast to most research, which has shown that reaction time is faster after caffeine consumption or a bout of aerobic exercise. Previous studies have found reaction time to be faster following exercise Davranche and McMorris [1] [4] [13]-[15] and caffeine consumption [7] [11] [16] [17]. Crowe, Leicht, and Spinks [18] examined the effects of caffeine consumption and anaerobic exercise, and found no differences in reaction time between groups which is consistent with the findings of the current study.

For the false alarms portion of the Contingent CPT, there was a significant time effect, but not an effect for condition. All conditions decreased from 5 minutes after exercise or quiet reading to 20 minutes after exercise or quiet reading, displaying a possible learning curve. The measurement of false alarms is a measure of selective inhibition because irrelevant stimuli must be inhibited in order to decrease the false alarms score. The lack of a condition effect in the current study is similar to other previous research which showed no differences in measures of selective inhibition [13] [19] In contrast to the results from the current study, several studies have shown an improvement between conditions after exercise [9] [20] [21] and Childs and de Wit [7] also saw an improvement in inhibition following the consumption of caffeine. Hogervorst et al. [10] also found greater inhibition in a group that received a combination of caffeine and exercise.

Once again this study showed differences by condition or time on performance of the WCST as measured by total errors. The WCST is used to measure of executive function, specifically planning, set shifting, working memory and goal directed behavior [11]. In contrast to the findings of the current study, previous research has found improvements in executive function following exercise [4] [22] and caffeine consumption [7] [17]. However, the findings of the current study are consistent with other studies which have not found a difference after caffeine consumption [11] [16].

The current study does have some limitations. The findings of the current study were based on a small number of participants, potentially influencing the non-significant findings. Though the number of participants in the 


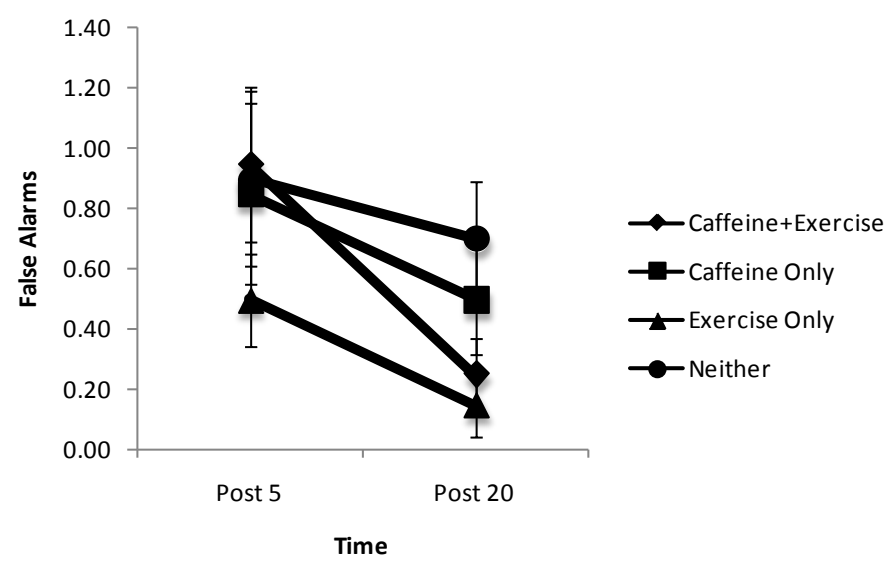

Figure 2. False alarms for contingent CPT by condition*time.

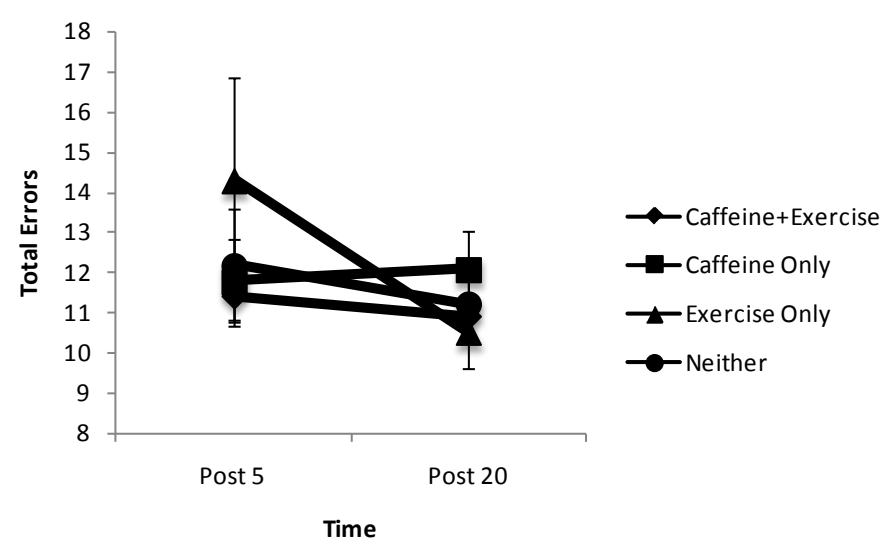

Figure 3. Total errors for Wisconsin card sort task by condition*time.

current study is similar to many of the previous research studies, it is possible that some of the results in the current study would have been statistically significant with a larger sample size. Another limitation may have come been the dosages of caffeine and exercise that were prescribed to the participants. Previous research has used a wide variety of intensities of exercise and dosages of caffeine. This study attempted to normalize caffeine and exercise based on the individual, but a lower intensity of exercise or higher dose of caffeine may have been better to show the differences hypothesized. A final limitation may have been the measures of cognitive function utilized in the present study. While the Contingent CPT and WCST are valid and reliable tests of cognitive function, other measures of cognitive function might be more sensitive to the influence of exercise and caffeine.

\section{Conclusion}

In this current study, there were no significant differences found for the three variables tested-total errors on the WCST, and reaction time and false alarms on the Contingent CPT, for condition effect, time effect or condition*time interaction, except for a significant time effect on false alarms on the Contingent CPT. There are many variables that may account for the variability in results seen in research studying at the effects of caffeine and exercise, individually and combined, on cognitive function. The lack of consistency points to the need for more research.

\section{Acknowledgements}

The authors would like to thank the Elon University Honors Program and Undergraduate Research Program for support which included funding for a Summer Undergraduate Research Experience. 


\section{References}

[1] Davranche, K. and McMorris, T. (2009) Specific Effects of Acute Moderate Exercise on Cognitive Control. Brain and Cognition, 69, 565-570. http://dx.doi.org/10.1016/j.bandc.2008.12.001

[2] Lambourne, K., Audiffren, M. and Tomporowski, P.D. (2010) Effects of Acute Exercise on Sensory and Executive Processing Tasks. Medicine \& Science in Sports \& Exercise, 42, 1396-1402. http://dx.doi.org/10.1249/mss.0b013e3181cbee11

[3] Tomporowski, P.D. (2003) Effects of Acute Bouts of Exercise on Cognition. Acta Psychologica, 112, $297-324$. http://dx.doi.org/10.1016/s0001-6918(02)00134-8

[4] Pontifex, M.B., Hillman, C.H., Fernhall, B., Thompson, K.M. and Valentini, T.A. (2009) The Effect of Acute Aerobic and Resistance Exercise on Working Memory. Medicine \& Science in Sports \& Exercise, 41, 927-934. http://dx.doi.org/10.1249/MSS.0b013e3181907d69

[5] Chang, Y.K., Labban, J.D., Gapin, J.I. and Etnier, J.L. (2012) The Effects of Acute Exercise on Cognitive Performance: A Meta-Analytic Review. Brain Research, 1453, 87-101. http://dx.doi.org/10.1016/j.brainres.2012.02.068

[6] Brunyé, T.T., Mahoney, C.R., Lieberman, H.R. and Taylor, H.A. (2010) Caffeine Modulates Attention Network Function. Brain and Cognition, 72, 181-188. http://dx.doi.org/10.1016/j.bandc.2009.07.013

[7] Childs, E. and De Wit, H. (2006) Subjective, Behavioral, and Physiological Effects of Acute Caffeine in Light, Nondependent Caffeine Users. Psychopharmacology, 185, 514-523. http://dx.doi.org/10.1007/s00213-006-0341-3

[8] Fine, B.J., Kobrick, J.L., Lieberman, H.R., Marlowe, B., Riley, R.H. and Tharion, W.J. (1994) Effects of Caffeine or Diphenhydramine on Visual Vigilance. Psychopharmacology, 114, 233-238. http://dx.doi.org/10.1007/BF02244842

[9] Hogervorst, E.E., Riedel, W.J., Kovacs, E.E., Brouns, F.F. and Jolles, J.J. (1999) Caffeine Improves Cognitive Performance after Strenuous Physical Exercise. International Journal of Sports Medicine, 20, 354-361. http://dx.doi.org/10.1055/s-2007-971144

[10] Hogervorst, E., Bandelow, S., Schmitt, J., Jentjens, R., Oliveira, M., Allgrove, J., Carter, T. and Gleeson, M. (2008) Caffeine Improves Physical and Cognitive Performance during Exhaustive Exercise. Medicine \& Science in Sports \& Exercise, 40, 1841-1851. http://dx.doi.org/10.1249/MSS.0b013e31817bb8b7

[11] Adan, A. and Serra-Grabulosa, J.M. (2010) Effects of Caffeine and Glucose, Alone and Combined, on Cognitive Performance. Human Psychopharmacology, 25, 310-317. http://dx.doi.org/10.1002/hup.1115

[12] Smid, H.G., de Witte, M.R., Homminga, I. and van den Bosch, R.J. (2006) Sustained and Transient Attention in the Continuous Performance Test. Journal of Clinical and Experimental Neuropsychology, 28, 859-883. http://dx.doi.org/10.1080/13803390591001025

[13] Davranche, K., Hall, B. and McMorris, T. (2009) Effect of Acute Exercise on Cognitive Control Required during an Eriksen Flanker Task. Journal of Sport \& Exercise Psychology, 31, 628-639.

[14] Pesce, C., Capranica, L., Tessitore, A. and Figura, F. (2002) Focusing of Visual Attention under Submaximal Physical Load. International Journal of Sport Psychology, 1, 275-292. http://dx.doi.org/10.1080/1612197X.2003.9671719

[15] Pesce, C., Tessitore, A., Casella, R. and Capranica, L. (2007) Focusing of Visual Attention at Rest and during Physical Exercise in Soccer Players. Journal of Sports Sciences, 25, 1259-1270. http://dx.doi.org/10.1080/02640410601040085

[16] Durlach, P.J. (1998) The Effects of a Low Dose of Caffeine on Cognitive Performance. Psychopharmacology, 140, 116-119. http://dx.doi.org/10.1007/s002130050746

[17] Giles, G.E., Mahoney, C.R., Brunyé, T.T., Gardony, A.L., Taylor, H.A. and Kanarek, R.B. (2012) Differential Cognitive Effects of Energy Drink Ingredients: Caffeine, Taurine and Glucose. Pharmacology Biochemistry and Behavior, 102, 569-577. http://dx.doi.org/10.1016/j.pbb.2012.07.004

[18] Crowe, M.J., Leicht, A.S. and Spinks, W.L. (2006) Physiological and Cognitive Responses to Caffeine during Repeated, High-Intensity Exercise. International Journal of Sport Nutrition and Exercise Metabolism, 16, 528-544.

[19] Hillman, C.H., Snook, E.M. and Jerome, G.J. (2003) Acute Cardiovascular Exercise and Executive Control Function. International Journal of Psychophysiology, 48, 307-314. http://dx.doi.org/10.1016/s0167-8760(03)00080-1

[20] Lichtman, S. and Poser, E.G. (1983) The Effects of Exercise on Mood and Cognitive Functioning. Journal of Psychosomatic Research, 27, 43-52. http://dx.doi.org/10.1016/0022-3999(83)90108-3

[21] Sibley, B.A., Etnier, J.L. and Le Masurier, G.C. (2006) Effects of an Acute Bout of Exercise on Cognitive Aspects of Stroop Performance. Journal of Sport \& Exercise Psychology, 28, 285-299.

[22] Tomporowski, P.D., Cureton, K., Armstrong, L.E., Kane, G.M., Sparling, P.B. and Millard-Stafford, M. (2005) ShortTerm Effects of Aerobic Exercise on Executive Processes and Emotional Reactivity. International Journal of Sport and Exercise Psychology, 3, 131-146. http://dx.doi.org/10.1080/1612197X.2005.9671763 